\title{
Sublingual Buprenorphine Efficacy in Renal Colic Pain Relief: A Randomized Placebo-Controlled Clinical Trial
}

Javad Mozafari - Kambiz Masoumi (D) Arash Forouzan •

Hassan Motamed · Malehi Amal Saki · Marzieh Dezham

Received: August 1, 2017 / Published online: October 20, 2017

(C) The Author(s) 2017. This article is an open access publication

\begin{abstract}
Introduction: This study was designed to compare the analgesic efficacy of sublingual (SL) buprenorphine and intravenous (IV) ketorolac for renal colic pain relief.

Methods: The present study was carried out as a double-blind, double-dummy randomized clinical trial in patients diagnosed with acute renal colic attending the emergency department. The study subjects were 63 patients with confirmed renal stones. The subjects were randomly divided into two groups. One group, which included 32 patients, received SL buprenorphine tablets $(2 \mathrm{mg})$ with an IV placebo, and another group, including 31 patients, received ketorolac tromethamine $(30 \mathrm{mg}$ ) with a SL placebo. After medication, the pain of subjects was measured by a standard visual pain analogous scale (VPAS)
\end{abstract}

Enhanced content To view enhanced content for this article go to http://www.medengine.com/Redeem/ 53CCF0604705E1C2.

J. Mozafari · K. Masoumi ( $₫)$ · A. Forouzan .

H. Motamed · M. Dezham

Department of Emergency Medicine, Ahvaz

Jundishapur University of Medical Sciences, Ahvaz,

Iran

e-mail: emdajums@yahoo.com

M. A. Saki

Department of Vital Statistics, Faculty of Health, Ahvaz Jundishapur University of Medical Sciences, Ahvaz, Iran in minutes $0,20,40$, and 60 of study. Also, probable adverse effects were recorded.

Results: In $28.1 \%$ of patients in the buprenorphine group, the mean pain score decreased from 9.2 to $5.9,2.8$, and 1.5 after 20,40 , and $60 \mathrm{~min}$, respectively, as determined by VPAS. Also, in $38.7 \%$ of patients in the ketorolac group, the mean pain score decreased from 9.1 to $5.5,3.0$, and 1.6 after 20,40 , and $60 \mathrm{~min}$, respectively, as determined by VPAS. The two groups did not significantly differ for pain reduction at 20,40, and $60 \mathrm{~min}(P$ value $=0.16$, 0.34 , and 0.3 , respectively). No adverse effects were seen in the ketorolac group, but vomiting (18.8\%), nausea (18.8\%), and dizziness (21.9\%) were detected in the buprenorphine group.

Conclusions: We found no difference between SL buprenorphine and intravenous ketorolac in renal colic pain relief but more adverse effects in the buprenorphine group.

Trial Registration Iranian Registry of Clinical trials identifier, IRCT2015041421773N1.

Keywords: Ketorolac; Pain relief; Renal colic; Sublingual buprenorphine; VPAS

\section{INTRODUCTION}

Pain is the most common reason that patients visit an emergency department (ED) [1]. Renal colic is known with severe pain and frequently encountered in EDs, with 5-15\% prevalence in 
the population [2]. Kidney stones cause pain by obstructing the uterus, and may lead to hydronephrosis [2]. The patient's pain relief places the responsibility on emergency physicians to find safe and effective methods of treatment [1]. Often, pain relief in renal colic patients is a challenge in ED, but the cornerstone of treatment includes narcotics and NSAIDs.

New effective analgesics have been studied for the treatment of pain. Buprenorphine is a partial opioid receptor agonist with opioid receptor antagonist properties. It has a high affinity and low-to-moderate intrinsic activity at the opioid receptor [3]. Buprenorphine is effective for the treatment of moderate to severe pain [4]. It also demonstrates the potential of sublingual opioids for the release of the baseline pain. It is a lipophilic molecule with poor oral bioavailability (approximately 10\%) and undergoes first-pass metabolism, but it has good sublingual bioavailability (approximately 50\%), with large intersubject variability [3]. Following its sublingual administration, there is no considerable rise in its plasma concentrations for $20 \mathrm{~min}$; the time to maximum concentration is variable, ranging from $40 \mathrm{~min}$ to $6 \mathrm{~h}$ [4]. Potency is high and serum concentrations are linearly related to dose (from 1 to $32 \mathrm{mg}$ ) [3].

Intravenous (IV) administration of either NSAIDs or opioids is the common route for pain relief in renal colic. Sometimes, this route is accompanied by adverse effects, and takes more time than the oral or sublingual route. It may be not suitable in overcrowded emergency wards. Furthermore, there are a few investigations about pain management by buprenorphine, especially for renal colic [5]. Therefore, this study was designed to compare the effectiveness of sublingual buprenorphine versus intravenous ketorolac tromethamine for pain relief in renal colic.

\section{METHODS}

A clinical trial, double-blind, double-dummy, randomized study was carried out in patients diagnosed with renal colic. The symptoms for inclusion criteria were colic flank pain associated with costo-vertebral angle tenderness and urinary tract irritation symptoms that suggest clinical diagnosis of renal colic based on history and physical examination and pain score greater than 3 , as determined by visual pain analogous scale (VPAS), attending emergency departments of Golestan general Hospital at Ahvaz, southwest of Iran, with 73,000 annual visits from August 2015 to April 2016.

\section{Exclusion Criteria}

Age $<18$ years and age $>55$ years, any pain killer during the previous $6 \mathrm{~h}$, addiction (self-report or medical record), history of allergic reaction to opioids or NSAIDs, systolic blood pressure $<90 \mathrm{mmHg}$, abdominal tenderness and rebound, body temperature $>38^{\circ} \mathrm{C}$, history or documents suggesting ischemic heart disease, liver disorder, respiratory disease, renal failure, gastrointestinal bleeding, active peptic ulcer, seizure, metabolic disorder, pregnancy, clinical concern for abdominal aortic aneurysm or dissection, inability to speak, and any intervention beyond the study protocol because of intolerable pain or patient disagreement.

\section{Study Design}

The subjects were randomly divided into two groups. After applying inclusion and exclusion criteria, one group received either $2 \mathrm{mg}$ sublingual buprenorphine tablet [Mehr darou Pharmaceutical Company, Razi distribution company; Tehran, Iran; 2 mg, Sublingual Tab] with $1 \mathrm{cc}$ intravenous sterile water as placebo simultaneously, and another group received $30 \mathrm{mg}$ ketorolac tromethamine [Caspian Tamin Pharmaceutical Company; Rasht, Iran; $30 \mathrm{mg} /$ cc, Ampule] with a sublingual tab similar to buprenorphine (made by the college pharmacy laboratory simultaneously; Ahvaz Jundishapur University of Medical Sciences) as placebo. Before intervention, and at 20,40, and $60 \mathrm{~min}$ after administration, pain scores were measured and recorded by a fixed emergency medicine attending consultant based on a standard 10-gauge VPAS $[0=$ no pain, $10=$ the worst pain $]$. 
At 40 min after administration, any patient with a pain score $>5$ or any patient with a pain score $>2$ in minute of $60 \mathrm{~min}$, received $1 \mu \mathrm{g} / \mathrm{kg}$ of intravenous fentanyl [Caspian Tamin Pharmaceutical Company; Rasht, Iran; Ampule, $0.5 \mathrm{mg} / 10 \mathrm{ml}]$ as rescue treatment.

During $1 \mathrm{~h}$ of intervention, if we needed to use any medicine apart from the study protocol, we excluded that patient from the study. After $60 \mathrm{~min}$, if renal colic pain continued or recurred, we used additional or other medications and hospitalized the patient in the urology department in order to make more evaluations based on their situation.

Both groups were monitored for adequate pain control and probable adverse effects, including respiratory depression (a respiratory rate below 12 breaths/min), hypotension (a drop of more than $20 \%$ in systolic blood pressure), nausea, vomiting, pruritus, headache, lightheadedness, and drowsiness, during study.

Also, just before, and 60 min after medication, vital signs and $\mathrm{O}_{2}$ saturation were recorded in each patient. Both groups were followed for presumptive complications until $24 \mathrm{~h}$ after medication. Baseline demographic characteristics of all patients were gathered, including gender, age, weight, past medical history, and family history. Additional interventions were recorded.

Acute renal colic because of renal stones was confirmed by clinical manifestations associated with urine analysis and ultrasonography or CT scanning. If renal stones were not confirmed, patients were excluded.

All authors confirmed that the study received the approval of an institutional review board at Ahvaz Jundishapur University of Medical Sciences (ethical number of AJUMS.REC.1393.433). All procedures followed were in accordance with the ethical standards of the responsible committee on human experimentation (institutional and national) and with the Helsinki Declaration of 1964, as revised in 2013. Informed consent was obtained from all patients for being included in the study.

\section{Data Analysis}

According to the study by Jalili et al. [6], the mean \pm SD of pain scores at $60 \mathrm{~min}$ is $2.2 \pm 0.7$. In addition, according to 0.6 difference in pain score, $\alpha=0.05$ and power of $80 \%$, the sample size in each group was 30 patients.

The independent Student's $t$ test was used to compare the baseline demographic characteristics and the effect of intervention on pain scores between the study groups at each time point. Adverse effects were compared using Chi-square tests, and the value of $p<0.05$ was regarded as statistically significant. Statistical analysis was achieved using dependent Student's $t$ test, which aimed to compare variation of vital signs before and after medication. Finally, data were analyzed using the Statistical Package for Social Science (SPSS) version 17.

\section{RESULTS}

Of 95 patients, 32 of them were excluded (Fig. 1). Of the 63 patients, 32 and 31 patients were assigned into buprenorphine and ketorolac groups, respectively. There were no significant differences in baseline characteristics between the two groups statistically (Table 1).

The comparison of pain scores over time in the two groups is shown in Table 2 and Fig. 2. The mean pain score in nine $(28.1 \%)$ of patients in the buprenorphine group (no need for rescue treatment) decreased from 9.2 to 5.9, 2.8 and 1.5 within 20, 40, and $60 \mathrm{~min}$, respectively, as determined by VPAS. Also, in 12 (38.7\%) patients in the ketorolac group (no need for rescue treatment), the mean pain score decreased from 9.1 to 5.5, 3.0 and 1.6 after 20, 40, and $60 \mathrm{~min}$, respectively, as determined by VPAS (Table 3 ).

There was no pain relief (less than a score of 5) at $40 \mathrm{~min}$ in 23 patients $(71.9 \%)$ in the buprenorphine group and 19 patients (61.3\%) in the ketorolac group; therefore fentanyl was used as rescue pain killer. The two regimens did not significantly differ for pain reduction $(P>0.05)$. No adverse effects were seen in the ketorolac group, but vomiting $(18.8 \%)$, nausea 


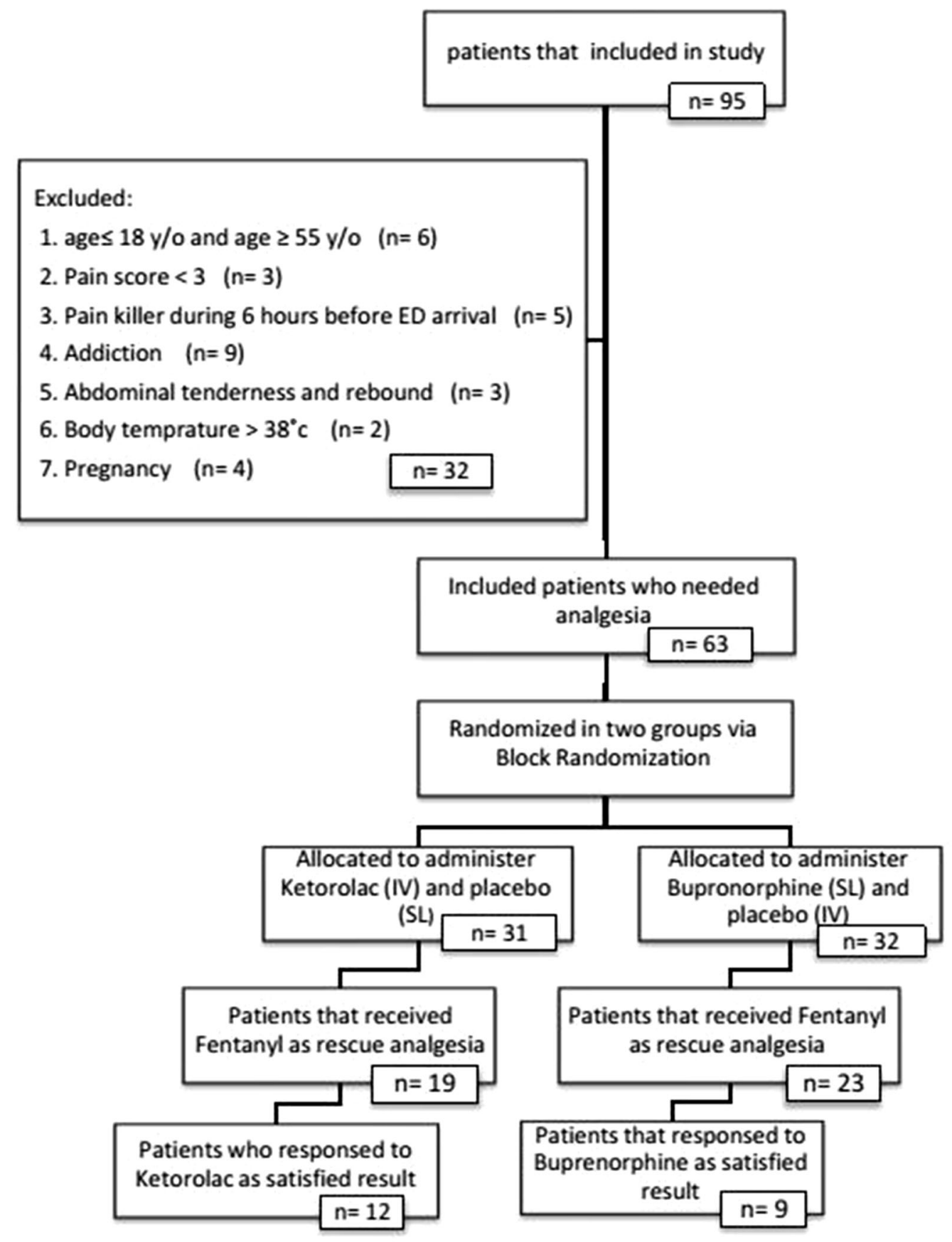

Fig. 1 Flowchart of the study

(18.8\%), and dizziness (21.9\%) were seen in patients of buprenorphine group (Table 4).

The vital signs of each group were not statistically different (Table 5).

\section{DISCUSSION}

Buprenorphine has been approved as an analgesic for various types of pain. Jakobs et al. showed that buprenorphine leads to 
Table 1 Baseline demographic characteristics of study patients

\begin{tabular}{lllll}
\hline Variables & Buprenephrine $(\boldsymbol{n}=\mathbf{3 2})$ & Ketorolac $(\boldsymbol{n}=\mathbf{3 1})$ & All patients & $\boldsymbol{P}$ value \\
\hline Male & $78.4 \%$ & $87.1 \%$ & $82.75 \%$ & 0.52 \\
Mean age $(\mathrm{year})^{\mathrm{a}}$ & $39.18 \pm 1.63$ & $35.58 \pm 2.04$ & $37.38 \pm 1.83$ & 0.17 \\
Mean weight $(\mathrm{kg})^{\mathrm{a}}$ & $72.43 \pm 1.43$ & $74.64 \pm 1.52$ & $73.53 \pm 1.47$ & 0.4 \\
Past medical history & $75 \%$ & $67.7 \%$ & $71.35 \%$ & 0.58 \\
Family history & $78.1 \%$ & $80.6 \%$ & $79.35 \%$ & 1.000 \\
\hline
\end{tabular}

${ }^{a}$ Values are mean $( \pm S D)$

Table 2 Comparison of pain severity (mean \pm SD) in two groups at different time points

\begin{tabular}{llllll}
\hline Time points & & $\boldsymbol{T}_{\mathbf{0}}$ & $\boldsymbol{T}_{\mathbf{2 0}}$ & $\boldsymbol{T}_{\mathbf{4 0}}$ & $\boldsymbol{T}_{\mathbf{6 0}}$ \\
\hline Groups & Buprenephrine group & $9.2 \pm 0.97$ & $5.9 \pm 1$ & $2.8 \pm 1.16$ & $1.55 \pm 0.52$ \\
& Ketorolac group & $9.1 \pm 0.99$ & $5.5 \pm 1.16$ & $3 \pm 1.28$ & $1.66 \pm 0.65$ \\
$P$ value & & 0.28 & 0.16 & 0.34 & 0.3 \\
\hline
\end{tabular}

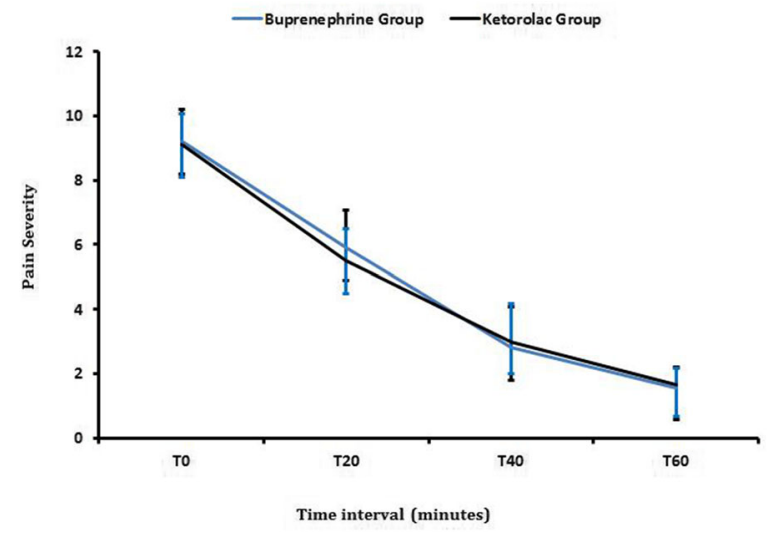

Fig. 2 Trend of pain score changes in buprenephrine and ketorolac groups $(P>0.05$, not significant $)$

significantly reduced pain scores in comparison to procaine in pancreatitis [7]. It is also used for treating various behavioral and psychiatric disorders [8], but there are few studies regarding pain management of acute renal colic by buprenorphine. In addition, some studies have indicated that ketorolac [8-10] is an effective analgesic for acute renal colic pain relief.

In our study, we found that $2 \mathrm{mg}$ of sublingual buprenorphine was as effective as $30 \mathrm{mg}$ intramuscular ketorolac for renal colic pain relief. Two agents showed a similar decreased trend. However, our results did not show any significant differences among the two groups over time.

Our results are also comparable with the results of Jalili et al. [6], who studied the effectiveness of $0.4 \mathrm{mg}$ sublingual buprenorphine and $5 \mathrm{mg}$ intravenous morphine in patients with acute extremity fractures. The pain scores were compared, and no significant difference was seen between the two groups. Also, they did not find any adverse effects in the buprenorphrine group. Payandemehr et al. [5] evaluated the efficacy of $2 \mathrm{mg}$ sublingual buprenorphine with $1 \mathrm{mg} / \mathrm{kg}$ intravenous morphine sulfate for pain management of acute renal colic. They have shown that $86.5 \%$ of patients in the buprenorphine group did not require rescue analgesia and sublingual buprenorphine can be used as an effective analgesic with minor side effects for acute pain relief in patients with renal colic, and its effectiveness is comparable to that of IV morphine. They reported that dizziness was the only prominent side effect in the buprenorphine group compared to the morphine group. Although in the present study, in the buprenorphine group, $28.1 \%$ of patients did not need rescue analgesia, and some adverse 
Table 3 Comparison of pain severity between responders of two groups

\begin{tabular}{llllll}
\hline & Group & $\boldsymbol{T}_{\mathbf{0}}$ & $\boldsymbol{T}_{\mathbf{2 0}}$ & $\boldsymbol{T}_{\mathbf{4 0}}$ & $\boldsymbol{T}_{\mathbf{6 0}}$ \\
\hline Responder (no need for rescue treatment) & Buprenephrine $(n=9)$ & 9.2 & 5.9 & 2.8 & 1.5 \\
& Ketorolac $(n=12)$ & 9.1 & 5.5 & 3 & 1.6 \\
\hline
\end{tabular}

Table 4 Frequency of adverse effects of two analgesia regimens in renal colic patients

\begin{tabular}{llll}
\hline Adverse effects & $\begin{array}{l}\text { Vomiting } \\
(\%)\end{array}$ & $\begin{array}{l}\text { Nausea } \\
(\%)\end{array}$ & $\begin{array}{l}\text { Dizziness } \\
(\%)\end{array}$ \\
\hline $\begin{array}{l}\text { Buprenorphine } \\
\text { group }\end{array}$ & 18 & 18 & 21.9 \\
$\begin{array}{l}\text { Ketorolac group } \\
\text { (\%) }\end{array}$ & 0 & 0 & 0 \\
\hline
\end{tabular}

effects including nausea (18\%), vomiting (18\%), and dizziness (21.9\%) were observed. The dose, drug form, and administration route of buprenorphine were similar in both studies, but there was no consistency between percentage pain relief and side effects of buprenorphine in the two trials; thus, the reason for this difference is unknown based on the current study.

There is wide inter-patient variability in the sublingual absorption of buprenorphine, but within subjects the variability is low. Both maximum serum concentration $\left(C_{\max }\right)$ and area under the curve (AUC) of buprenorphine have positive linear relationships with dose of buprenorphine, although the increase is not directly dose-proportional. Maybe this characteristic of buprenorphine can explain the difference of pain relief in various studies.
Common side effects following buprenorphine administration may include sedation, nausea, vomiting, dizziness, headache, and respiratory depression. However, as a mu-opioid partial agonist with a demonstrated ceiling on respiratory depression, buprenorphine may have a better safety profile compared to a full mu agonist [4]. Buprenorphine side effects may be because of the other components inside the buprenorphine tablets (lactose monohydrate, mannitol, povidone, anhydrous citric acid, sodium citrate, butylated hydroxyanisole, corn starch, pregelatinized starch, and magnesium stearate) or higher dose of buprenorphine.

There is a potential limitation of buprenorphine in the setting of severe pain: its purported analgesic ceiling. Preclinical studies of buprenorphine indicated that it exhibits an analgesic ceiling or even a bell-shaped dose-response curve, where in doses exceeding the maximal analgesic dose were associated with decreased analgesic efficacy. More recent work has found such dose-response curves in some pain models but not in others [3]. The limitation of this study was its small sample size. So, to find any real difference in efficacy between two drugs.

Table 5 Vital signs of study patients in both groups before and after medication

\begin{tabular}{|c|c|c|c|c|c|c|}
\hline \multirow[t]{2}{*}{ Vital signs } & \multicolumn{3}{|c|}{ Buprenephrine group } & \multicolumn{3}{|c|}{ Ketorolac group } \\
\hline & $\overline{T_{0}}$ & $T_{40}$ & $P$ value & $\overline{T_{0}}$ & $T_{40}$ & $P$ value \\
\hline Systolic pressure & $133.9 \pm 2.8$ & $131.2 \pm 2.4$ & 0.35 & $127.7 \pm 2.9$ & $125.2 \pm 2$ & 0.29 \\
\hline Diastolic pressure & $82.8 \pm 1.43$ & $81.9 \pm 1.3$ & 0.52 & $83.06 \pm 1.7$ & $80.9 \pm 1.2$ & 0.11 \\
\hline $\mathrm{RR}$ & $16 \pm 0.46$ & $16.8 \pm 0.41$ & 0.09 & $16.9 \pm 0.40$ & $16.8 \pm 0.47$ & 0.93 \\
\hline $\mathrm{HR}$ & $81.7 \pm 1.6$ & $83.3 \pm 1.2$ & 0.1 & $83.8 \pm 1.7$ & $82.9 \pm 1.5$ & 0.52 \\
\hline $\mathrm{O}_{2} \mathrm{Sat} \%$ & $98.3 \pm 0.3$ & $99.1 \pm 0.23$ & 0.001 & $98.2 \pm 0.3$ & $98.8 \pm 0.3$ & 0.05 \\
\hline
\end{tabular}

$R R$ respiratory rate, $H R$ heart rate

*Values are mean $( \pm S D)$ 


\section{LIMITATIONS}

The limitation of current study is that we would like to compare pain scores over time between responders (subjects who did not require fentanyl) of buprenorphine group (28.1\%) and ketorolac group (38.7\%). We could not conduct this analysis due to a small number of responders in each group (nine and 12 patients in buprenorphine and ketorolac groups, respectively).

\section{CONCLUSIONS}

The results of this study indicate that although ketorolac was more effective than buprenorphine (ketorolac 38.7\% versus buprenorphine $28.1 \%)$, there was no significant difference between two analgesic regimens in acute renal colic pain management. Buprenorphine has not been as extensively studied in certain populations (e.g., in individuals suffering from pain of malignant origin) as other opioid analgesics, and additional research is needed to better define the role of buprenorphine in various patient subpopulations.

\section{ACKNOWLEDGEMENTS}

No funding or sponsorship was received for this study or publication of this article. All named authors meet the International Committee of Medical Journal Editors (ICMJE) criteria for authorship for this manuscript, take responsibility for the integrity of the work as a whole, and have given final approval to the version to be published.

The authors are grateful to the ED executive staff of Golestan hospital, Jundishapur University of Medical Sciences, Ahvaz, Iran, for their assistance and would also like to thank all patients who participated in this study.

Disclosures. Javad Mozaffari, Kambiz Masoumi, Arash Forouzan, Hassan Motamed, Malehi Amal Saki, and Marzieh Dezham have nothing to disclose.
Compliance with Ethics Guidelines. All authors confirm that this study received the approval of an institutional review board at Ahvaz Jundishapur University of Medical Sciences (ethical number of AJUMS.REC.1393.433). All procedures followed were in accordance with the ethical standards of the responsible committee on human experimentation (institutional and national) and with the Helsinki Declaration of 1964, as revised in 2013. Informed consent was obtained from all patients for being included in the study.

Data Availability. The datasets generated during and/or analyzed during the current study are available from the corresponding author on reasonable request.

Open Access. This article is distributed under the terms of the Creative Commons Attribution-NonCommercial 4.0 International License (http://creativecommons.org/licenses/ by-nc/4.0/), which permits any noncommercial use, distribution, and reproduction in any medium, provided you give appropriate credit to the original author(s) and the source, provide a link to the Creative Commons license, and indicate if changes were made.

\section{REFERENCES}

1. Hesse A, Brändle E, Wilbert D, Köhrmann KU, Alken P. Study on the prevalence and incidence of urolithiasis in Germany comparing the years 1979 vs. 2000. Eur Urol. 2003;44(6):709-13.

2. Marx J, Walls R, Hockberger R. Rosen's emergency medicine-concepts and clinical practice, vol. 2. 8th ed. Elsevier Health Sciences; 2013. p. 1336-42.

3. Reisfield GM, Wilson GR. Rational use of sublingual opioids in palliative medicine. J Palliat Med. 2007;10(2):465-75.

4. Johnson RE, Fudala PJ, Payne R. Buprenorphine: considerations for pain management. J Pain Symptom Manage. 2005;29(3):297-326.

5. Payandemehr P, Jalili M, Davani BM, Dehpour AR. Sublingual buprenorphine for acute renal colic pain management: a double-blind, randomized controlled trial. Int J Emerg Med. 2014;7(1):1. 
6. Jalili M, Fathi M, Moradi-Lakeh M, Zehtabchi S. Sublingual buprenorphine in acute pain management: a double-blind randomized clinical trial. Ann Emerg Med. 2012;59(4):276-80.

7. Jakobs R, Adamek MU, Von Bubnoff AC, Riemann JF. Buprenorphine or procaine for pain relief in acute pancreatitis A prospective randomized study. Scand J Gastroenterol Suppl. 2000;35(12):1319-23.

8. Pincus S, MacBean C, Taylor D. The effects of temperature, age and sex on presentations of renal colic in Melbourne. Aust. Eur J Emerg Med. 2010;17(6):328-31.
9. Kolasani BP, Juturu J. Intramuscular ketorolac versus diclofenac in acute renal colic: a comparative study of efficacy and safety. Indian J App Basic Med Res. 2013;2(8):923-31.

10. Tanabe P, Buschmann M. A prospective study of ED pain management practices and the patient's perspective. J Emerg Nurs. 1999;25(3):171-7. 